\title{
Lie Ideals and Jordan Triple Derivations in Rings
}

\author{
Motoshi Hongan (*) - Nadeem ur Rehman (**) - Radwan Mohammed \\ $\operatorname{AL-OMARY}(* *)$
}

ABSTRACT - In this paper we prove that on a 2-torsion free semiprime ring $R$ every Jordan triple (resp. generalized Jordan triple) derivation on Lie ideal $L$ is a derivation on $L$ (resp. generalized derivation on $L)$.

\section{Introduction.}

This paper is motivated by the work of Jing and $\mathrm{Lu}$ [13]. Throughout the present paper, $R$ will denote an associative ring with center $Z(R)$. A ring $R$ is said to be 2 -torsion free, if whenever $2 x=0$ with $x \in R$, implies $x=0$. Recall that $R$ is prime if for any $a, b \in R, a R b=\{0\}$ implies that $a=0$ or $b=0$, and $R$ is semiprime if for all $a \in R$, $a R a=\{0\}$ implies $a=0$. An additive subgroup $L$ of $R$ is said to be a Lie ideal of $R$ if $[L, R] \subseteq L$. A Lie ideal $L$ is said to be square-closed if $a^{2} \in L$ for all $a \in L$. An additive mapping $\delta: R \longrightarrow R$ is called a derivation (resp. a Jordan derivation) if $(x y)^{\delta}=x^{\delta} y+x y^{\delta}\left(\operatorname{resp} .\left(x^{2}\right)^{\delta}=x^{\delta} x+x x^{\delta}\right)$ holds for all $x, y \in R$. A famous result due to Herstein [11, Theorem 3.3] states that a Jordan derivation of a prime ring of characteristic not equal to 2 must be a derivation. A brief proof of Herstein's result can also be found in [8]. This result was extended to 2-torsion free semiprime rings by Cusack [10] and subsequently, by Bresar [7] .

Following [6], an additive mapping $\delta: R \longrightarrow R$ is called a Jordan triple derivation if $(x y x)^{\delta}=x^{\delta} y x+x y^{\delta} x+x y x^{\delta}$ holds for all $x, y \in R$. One can

(*) Indirizzo dell'A.: Department of Mathematics, Tsuyama College of Technology, 624-1 Numa, Tsuyama, Okayama (Japan).

E-mail: hongan@mx32.tiki.ne.jp

(**) Indirizzo dell'A.: Department of Mathematics, Aligarh Muslim University, Aligarh 202002 (India).

E-mail: rehman100@gmail.com, radwan959@yahoo.com

This research is supported by UGC, India, Grant No. 36-8/2008(SR). 
148 Motoshi Hongan - Nadeem ur Rehman - Radwan Mohammed AL-Omary

easily prove that any Jordan derivation on an arbitrary 2-torsion free ring is a Jordan triple derivation (see for example [8] where further references can be found). Bresar has proved the following result.

THEOREM 1.1 (6, Theorem 4.3). Let $R$ be a 2-torsion free semiprime ring and let $\delta: R \rightarrow R$ be a Jordan triple derivation. In this case $\delta$ is a derivation.

An additive mapping $F: R \longrightarrow R$ is said to be a generalized derivation (resp. a generalized Jordan derivation) on $R$ if there exists a derivation $\delta: R \longrightarrow R$ such that $(x y)^{F}=x^{F} y+x y^{\delta}$ (resp. $\left.\left(x^{2}\right)^{F}=x^{F} x+x x^{\delta}\right)$ holds for all $x, y \in R$. An additive mapping $F: R \longrightarrow R$ is said to be a generalized Jordan triple derivation on $R$ if there exists a Jordan triple derivation $\delta: R \longrightarrow R$ such that $(x y x)^{F}=x^{F} y x+x y^{\delta} x+x y x^{\delta}$ holds for all $x, y \in R$. Motivated by the above result Jing and Lu have proved the following generalization of Theorem 1.1.

THEOREM 1.2 (13, Theorem 3.5). Let $R$ be a 2-torsion free prime ring, then every generalized Jordan triple derivation on $R$ is a generalized derivation.

Very recently, Vukman [18] extended the result mentioned above for 2torsion free semiprime rings. In the present paper, our objective is to generalize Theorem 1.1 and 1.2 on Lie ideal of $R$.

\section{Jordan Triple Derivations.}

It is obvious to see that evey derivation is a Jordan triple derivation, but the converse need not to be true in general. Motivated by the Theorem 1.1, in the present section, it is shown that on a 2-torsion free semiprime ring $R$ every Jordan triple derivation on Lie ideal $L$ is a derivation on $L$. More precisely, we prove the following:

THEOREM 2.1. Let $R$ be a 2-torsion free semiprime ring and $L \nsubseteq Z(R)$ be a square-closed Lie ideal of $R$. If an additive mapping $\delta: R \longrightarrow R$ satisfies

$$
(a b a)^{\delta}=a^{\delta} b a+a b^{\delta} a+a b a^{\delta} \text { for all } a, b \in L
$$

and $L^{\delta} \subseteq L$, then $\delta$ is a derivation on $L$. 
To facilitate our discussion, we shall begin with the following lemmas:

LEMMa 2.1 (4, Lemma 4). Let $L \nsubseteq Z(R)$ be a Lie ideal of a 2-torsion free prime ring $R$ and $a, b \in R$. If $a L b=\{0\}$, then $a=0$ or $b=0$.

Lemma 2.2 (17, Lemma 2.4). Let $R$ be a 2-torsion free semiprime ring, $L$ be a Lie ideal of $R$ such that $L \nsubseteq Z(R)$ and let $a \in L$. If $a L a=\{0\}$, then $a^{2}=0$ and there exisits a nonzero ideal $K=R[L, L] R$ of $R$ generated by $[L, L]$ such that $[K, R] \subseteq L$ and $K a=a K=\{0\}$.

COROLLARY 2.1. Let $R$ be a 2-torsion free semiprime ring, $L$ be a Lie ideal of $R$ such that $L \nsubseteq Z(R)$ and let $a, b \in L$.

(1) If $a L a=\{0\}$, then $a=0$.

(2) If $a L=\{0\}$ (or $L a=\{0\}$ ), then $a=0$.

(3) If $L$ is square-closed, and $a L b=\{0\}$, then $a b=0$ and $b a=0$.

Proof. (1) Since $K a=R[L, L] R a=\{0\}$ and $a^{2}=0$ by Lemma 2.2, we have $0=[[a, x], a] y a=$ axaya for all $x, y \in R$, and so, we have axayaxa $=0$. Since $R$ is semiprime, we get $a x a=0$ for all $x \in R$. Moreover, by semiprimeness of $R$, we get $a=0$.

(2) It is clear by (1).

(3) If $a L b=\{0\}$, then we have $b a L b a=\{0\}$ obviously, and so we have $b a=0$ by (1). Moreover, since $a b L a b \subseteq a L b=\{0\}$, we get $a b=0$.

LEMMa 2.3 (17, Lemma 2.5). Let $R$ be a 2-torsion free ring, $L$ be a Lie ideal of $R$ and let $a, b \in L$. If $a u b+b u a=0$ for all $u \in L$, then aubLaub $=\{0\}$.

Lemma 2.4 (17, Lemma 2.7). Let $G_{1}, G_{2}, \cdots, G_{n}$ be additive groups, $R$ a 2-torsion free semiprime ring and $L \nsubseteq Z(R)$ be a Lie ideal of $R$. Suppose that mappings $S: G_{1} \times G_{2} \times \cdots \times G_{n} \longrightarrow R$ and $T: G_{1} \times G_{2} \times \cdots \times G_{n} \longrightarrow R$ are addtitive in each argument. If $S\left(a_{1}, a_{2}, \cdots, a_{n}\right) x T\left(a_{1}, a_{2}, \cdots, a_{n}\right)=0$ for all $x \in L, a_{i} \in G_{i} i=1,2, \cdots n$, then $S\left(a_{1}, a_{2}, \cdots, a_{n}\right) x T\left(b_{1}, b_{2}, \cdots, b_{n}\right)=0$ for all $x \in L, a_{i}, b_{i} \in G_{i} i=1,2, \cdots n$.

Lemma 2.5. Let $\delta$ be a Jordan triple derivation and $L$ be a Lie ideal of R. For arbitrary $a, b, c \in L$, we have

$$
(a b c+c b a)^{\delta}=a^{\delta} b c+a b^{\delta} c+a b c^{\delta}+c^{\delta} b a+c b^{\delta} a+c b a^{\delta} .
$$


150 Motoshi Hongan - Nadeem ur Rehman - Radwan Mohammed AL-Omary

Proof. We have

$$
(a b a)^{\delta}=a^{\delta} b a+a b^{\delta} a+a b a^{\delta} \text { for all } a, b \in L .
$$

We compute $W=\{(a+c) b(a+c)\}^{\delta}$ in two different ways. On one hand, we find that $W=(a+c)^{\delta} b(a+c)+(a+c) b^{\delta}(a+c)+(a+c) b(a+c)^{\delta}$, and on the other hand $W=(a b a)^{\delta}+(a b c+c b a)^{\delta}+(c b c)^{\delta}$. Comparing two expressions, we obtain the required result.

REMARK 2.1. It is easy to see that every Jordan derivation of a 2torsion free ring satisfies (2.1) ( see [1] for reference).

For the purpose of this section, we shall write; $\Delta(a b c)=(a b c)^{\delta}$ $-a^{\delta} b c-a b^{\delta} c-a b c^{\delta}$, and $\Lambda(a b c)=a b c-c b a$. We list a few elementary properties of $\delta$ and $\Lambda$ :

(i) $\Delta(a b c)+\Delta(c b a)=0$

(ii) $\Delta((a+b) c d)=\Delta(a c d)+\Delta(b c d)$ and $\Lambda((a+b) c d)=\Lambda(a c d)+\Lambda(b c d)$

(iii) $\Delta(a(b+c) d)=\Delta(a b d)+\Delta(a c d)$ and $\Lambda(a(b+c) d)=\Lambda(a b d)+\Lambda(a c d)$

(iv) $\Delta(a b(c+d))=\Delta(a b c)+\Delta(a b d)$ and $\Lambda(a b(c+d))=\Lambda(a b c)+\Lambda(a b d)$

Proposition 2.2. Let $R$ be a 2-torsion free semiprime ring and $L \nsubseteq Z(R)$ be a square closed Lie ideal of $R$. If $\Delta(a b c)=0$ holds for all $a, b, c \in L$, then $\delta$ is a derivation of $L$.

Proof. Let assume $\Delta(a b c)=0$ for all $a, b, c \in L$, that is,

$$
(a b c)^{\delta}=a^{\delta} b c+a b^{\delta} c+a b c^{\delta} .
$$

Let $M=a b x a b$. We have

$$
\begin{aligned}
M^{\delta} & =\{a(b x a) b\}^{\delta}=(a)^{\delta}(b x a) b+a b^{\delta} x a b+a b x^{\delta} a b \\
& +(a b x) a^{\delta} b+(a b x a) b^{\delta} \text { for all } x, a, b \in L .
\end{aligned}
$$

On the other hand,

$$
M^{\delta}=\{(a b) x(a b)\}^{\delta}=(a b)^{\delta}(x a b)+(a b) x^{\delta}(a b)+(a b x)(a b)^{\delta} .
$$

Comparing (2.3) with (2.2) we get

$$
\left\{(a b)^{\delta}-a^{\delta} b-a b^{\delta}\right\}(x a b)+(a b x)\left\{(a b)^{\delta}-a^{\delta} b-a b^{\delta}\right\}=0
$$

that is, $a^{b} x a b+a b x a^{b}=0$, where $a^{b}$ stands for $(a b)^{\delta}-a^{\delta} b-a b^{\delta}$. Thus, by Lemma 2.3 we find that $\left(a^{b} x a b\right) y\left(a^{b} x a b\right)=0$ for all $a, b, x, y \in L$. Now, by 
Corollary 2.1, we obtain $a^{b} x a b=0$ for all $a, b, x \in L$. Now by Lemma 2.4, we obtain $a^{b} x c d=0$ for all $a, b, c, d, x \in L$. Hence, by using Corollary 2.1, we get $a^{b}=0$ for all $a, b \in L$ that is, $\delta$ is a derivation on $L$.

Lemma 2.6. Let L be a Lie ideal of $R$. For any $a, b, c, x \in L$, we have

$$
\Delta(a b c) x \Lambda(a b c)+\Lambda(a b c) x \Delta(a b c)=0 .
$$

Proof. For any $a, b, c, x \in L$, suppose that $N=a b c x c b a+c b a x a b c$. Now we find

$$
\begin{aligned}
N^{\delta}= & \{a(b c x c b) a+c(b a x a b) c\}^{\delta}=\{a(b c x c b) a\}^{\delta}+\left\{(c(b a x a b) c\}^{\delta}\right. \\
= & a^{\delta} b c x c b a+a b^{\delta} c x c b a+a b c^{\delta} x c b a \\
& +a b c x \delta c b a+a b c x c^{\delta} b a+a b c x c b^{\delta} a \\
& +a b c x c b a^{\delta}+c^{\delta} b a x a b c+c b^{\delta} a x a b c \\
& +c b a^{\delta} x a b c+c b a x^{\delta} a b c+c b a x a^{\delta} b c \\
& +c b a x a b^{\delta} c+c b a x a b c^{\delta} .
\end{aligned}
$$

On the other hand, we have

$$
\begin{aligned}
N^{\delta}= & \{(a b c) x(c b a)+(c b a) x(a b c)\}^{\delta} \\
= & (a b c)^{\delta} x(c b a)+(a b c) x^{\delta}(c b a)+(a b c x)(c b a)^{\delta} \\
& +(c b a)^{\delta}(x a b c)+(c b a) x^{\delta}(a b c)+(c b a x)(a b c)^{\delta} .
\end{aligned}
$$

By comparing last two expressions, we get

$$
-\Delta(c b a)(x c b a)+\Delta(c b a)(x a b c)+(a b c x) \Delta(c b a)-(c b a x) \Delta(c b a)=0 .
$$

This implies that $\Delta(a b c) x \Lambda(a b c)+\Lambda(a b c) x \Delta(a b c)=0$ for all $a, b, c \in L$.

LEMMA 2.7. Let $R$ be a semiprime ring and $L \nsubseteq Z(R)$ be a square closed Lie ideal of $R$. Then $\Delta(a b c) x \Lambda(r s t)=0$ holds for all $a, b, c, r, s, t, x \in L$.

Proof. By Lemma 2.6 we have $\Delta(a b c) x \Lambda(a b c)+\Lambda(a b c) x \Delta(a b c)=0$ for all $a, b, c, x \in L$. Thus, we get $\Delta(a b c) x \Lambda(a b c) L \Delta(a b c) x \Lambda(a b c)=\{0\}$ by Lemma 2.3, and hence we obtain $\Delta(a b c) x \Lambda(a b c)=0$, for all $a, b, c, x \in L$ by Corollary 2.1. Now, we find that $\Delta(a b c) x \Lambda(r s t)=0$, for all $a, b, c, r, s, t, x \in L$ by Lemma 2.4 .

LEMMA 2.8. Let $R$ be a 2-torsion free semiprime ring and $L$ be a square closed Lie ideal of $R$. If $\Lambda(a b c)=0$ for all $a, b, c \in L$, then $L \subseteq Z(R)$.

Proof. Assume that $L \nsubseteq Z(R)$. We have $\Lambda(a b c)=0$ for all $a, b, c \in L$, that is, $a b c=c b a$. Replacing $b$ by $2 t b$, we get $2 a t b c=2 c t b a$ for all 
152 Motoshi Hongan - Nadeem ur Rehman - Radwan Mohammed AL-Omary

$a, b, c, t \in L$. Again replacing $t$ by $2 t w$ and using the fact that $R$ is 2 -torsion free, we get $a t w b c=c t w b a$ and hence $a(t w b) c=a(b w t) c=a(b c) t w=a w t b c$. Thus, we find that $a[t, w] b c=0$ for all $a, b, c, t, w \in L$. By Corollaray 2.1, we get $[t, w]=0$ for all $t, w \in L$, that is, $L$ is a commutative Lie ideal of $R$. And so, we have $[a,[a, t]]=0$ for all $t \in R$ and hence by Sublemma on page 5 of [11], $a \in Z(R)$. Hence $L \subseteq Z(R)$, a contradiction. This completes the proof of the Lemma.

Proof of Theorem 2.1. Suppose $\delta: R \longrightarrow L$ is a Jordan triple derivation on $L$. Our goal will be to show that $\delta$ is a derivation of associative triple systems. We know that $\Delta(a b c)=-\Delta(c b a)$. Hence

$$
\begin{aligned}
2 \Delta(a b c) x \Delta(a b c)= & \Delta(a b c) x\{\Delta(a b c)-\Delta(c b a)\} \\
= & \Delta(a b c) x\left\{\Lambda(a b c)^{\delta}+\Lambda\left(c^{\delta} b a\right)\right. \\
& \left.+\Lambda\left(c b^{\delta} a\right)+\Lambda\left(c b a^{\delta}\right)\right\} .
\end{aligned}
$$

By Lemma 2.7, the above relation reduces to

$$
2 \Delta(a b c) x \Delta(a b c)=\Delta(a b c) x \Lambda(a b c)^{\delta}
$$

Similarly we obtain

$$
2 \Delta(a b c) x \Delta(a b c)=\Lambda(a b c)^{\delta} x \Delta(a b c)
$$

Now we have

$$
\begin{aligned}
0= & \{\Delta(a b c) x \Lambda(a b c)+\Lambda(a b c) x \Delta(a b c)\}^{\delta} \\
= & \Delta(a b c)^{\delta} x \Lambda(a b c)+\Delta(a b c) x^{\delta} \Lambda(a b c) \\
& +\Delta(a b c) x \Lambda(a b c)^{\delta}+\Lambda(a b c)^{\delta} x \Delta(a b c) \\
& +\Lambda(a b c) x^{\delta} \Delta(a b c)+\Lambda(a b c) x \Delta(a b c)^{\delta}
\end{aligned}
$$

and according to Lemma 2.6 and (2.4), and (2.5), we get

$$
0=4 \Delta(a b c) x \Delta(a b c)+\Delta(a b c)^{\delta} x \Lambda(a b c)+\Lambda(a b c) x \Delta(a b c)^{\delta} .
$$

We multiply the relation above from left by $\Delta(a b c) x \Delta(a b c) y$ for all $a, b, c, x$, $y \in L$ and by Lemma 2.7 we obtain $4 \Delta(a b c) x \Delta(a b c) y \Delta(a b c) x \Delta(a b c)=0$ for all $a, b, c, x, y \in L$. Since $R$ is a 2-torsion free, it follows that $\Delta(a b c) x \Delta(a b c) y \Delta(a b c) x \Delta(a b c)=0$, that is, $\Delta(a b c) x \Delta(a b c) L \Delta(a b c) x \Delta(a b c)=\{0\}$. By Corollary 2.1, we find that $\Delta(a b c) x \Delta(a b c)=0$ and again using Corollary 2.1, we find that $\Delta(a b c)=0$ for all $a, b, c \in L$ and hence by Proposion 2.1, we get the required result. 
THEOREM 2.2. Let $R$ be a 2-torsion free prime ring and $L \nsubseteq Z(R)$ be a nonzero square-closed Lie ideal of $R$. If an additive mapping $\delta: R \longrightarrow L$ satisfies that

$$
(a b a)^{\delta}=a^{\delta} b a+a b^{\delta} a+a b a^{\delta} \text { for all } a, b \in L,
$$

then $\delta$ is a derivation on $L$.

Proof. By Lemma 2.7, we have $\Delta(a b c) x \Lambda(r s t)=0$ for all $a, b, c$, $r, s, t, x \in L$, that is, $\Delta(a b c) L \Lambda(r s t)=\{0\}$. Now, either $\Delta(a b c)=0$ or $\Lambda(r s t)=0$ by Lemma 2.1. If $\Lambda(r s t)=0$ for all $r, s, t \in L$, then we get $L \subseteq Z(R)$ by Lemma 2.8, a contradiction. On the other hand if $\Delta(a b c)=0$ for all $a, b, c \in L$, then we get the required result by Proposition 2.1.

\section{Generalized Jordan Triple Derivations.}

An additive mapping $\mu: R \rightarrow R$ is called a Jordan triple left centralizer on $L$ if $(a b a)^{\mu}=a^{\mu} b a$ for all $a, b \in L$, and called a Jordan left centralizer on $L$ if $\left(a^{2}\right)^{\mu}=a^{\mu} a$ for all $a \in L$.

THEOREM 3.1. Let $R$ be a 2 -torsion free semiprime ring and $L \nsubseteq Z(R)$ be a square-closed Lie ideal. If $\mu: R \rightarrow R$ is a Jordan triple left centralizer on $L$, then $\mu$ is a Jordan left centralizer on $L$.

Proof. By the hypothesis, we have

$$
(a b a)^{\mu}=a^{\mu} b a \text { for all } a, b \in L .
$$

Replacing $a$ by $a+c$ in (3.1), we find that

$$
\{(a+c) b(a+c)\}^{\mu}=a^{\mu} b a+c^{\mu} b a+a^{\mu} b c+c^{\mu} b c \text { for all } a, b, c \in L .
$$

On the other hand, we obtain

$$
\{(a+c) b(a+c)\}^{\mu}=(a b c+c b a)^{\mu}+a^{\mu} b c+c^{\mu} b a \text { for all } a, b, c \in L .
$$

Combining last two expressions, we get

$$
(a b c+c b a)^{\mu}=a^{\mu} b c+c^{\mu} b a \text { for all } a, b, c \in L .
$$

Now replacing $c$ by $a^{2}$ in (3.2), we get

$$
\left(a b a^{2}+a^{2} b a\right)^{\mu}=a^{\mu} b a^{2}+\left(a^{2}\right)^{\mu} b a .
$$


154 Motoshi Hongan - Nadeem ur Rehman - Radwan Mohammed AL-Omary

Again replacing $b$ by $a b+b a$ in (3.1), we get

$$
\left(a b a^{2}+a^{2} b a\right)^{\mu}=a^{\mu} a b a+a^{\mu} b a^{2} .
$$

Combining (3.3) and (3.4), we obtain

$$
\left\{\left(a^{2}\right)^{\mu}-a^{\mu} a\right\} b a=0 \text { for all } a, b \in L .
$$

By setting $\Omega^{a}=\left(a^{2}\right)^{\mu}-a^{\mu} a$, we have

$$
\Omega^{a} b a=0 \text { for all } a, b \in L .
$$

Thus, by Corollary 2.1, we obtain

$$
\Omega^{a} a=0=a \Omega^{a} \text { for all } a \in L .
$$

Linearizing (3.6), we get

$$
\Omega^{a+b} a+\Omega^{a+b} b=0 \text { for all } a, b \in L .
$$

Now, we compute $\Omega^{a+b}=\left\{(a b+b a)^{\mu}-a^{\mu} b-b^{\mu} a\right\}+\left(a^{2}\right)^{\mu}-a^{\mu} a+$ $+\left(b^{2}\right)^{\mu}-b^{\mu} b$ and hence we have

$$
\Omega^{a+b}=\Phi(a, b)+a^{\mu}+b^{\mu} \text { for all } a, b \in L,
$$

where $\Phi(a, b)=(a b+b a)^{\mu}-a^{\mu} b-b^{\mu} a$. Thus, in view of (3.8), the expression (3.7) implies that

$$
\Omega^{a} b+\Phi(a, b) a+\Omega^{b} a+\Phi(a, b) b=0 .
$$

Again, replacing $a$ by $-\alpha$ in (3.9), we get

$$
\Omega^{a} b+\Phi(a, b) a-\Omega^{b} a-\Phi(a, b) b=0 .
$$

Adding (3.9) with (3.10), we find that

$$
\Omega^{a} b+\Phi(a, b) a=0 .
$$

On right multiplication of (3.11) by $\Omega^{a}$, we obtain

$$
0=\Omega^{a} b \Omega^{a}+\Phi(a, b) a \Omega^{a}=\Omega^{a} b \Omega^{a} .
$$

Thus, we obtain $\Omega^{a}=0$ for all $a \in L$ by Corollary 2.1. This gives that $\mu$ is a Jordan left centralizer.

THEOREM 3.2. Let $R$ be a 2-torsion free semiprime ring and $L \nsubseteq Z(R)$ be a square-closed Lie ideal. If $F: R \rightarrow R$ is a generalized Jordan triple derivation on $L$ with a Jordan triple derivation $\delta$ such that $L^{\delta} \subseteq L$, then $F$ is a generalized Jordan derivation on $L$. 
Proof. Since $F$ is a generalized Jordan triple derivation on $L$. Therefore we have

$$
(a b a)^{F}=a^{F} b a+a b^{\delta} a+a b a^{\delta} \text { for all } a, b \in L .
$$

In (3.12), we take $\delta$ as a Jordan triple derivation on $L$. Since $R$ is a 2-torsion free semiprime ring, so in view of Theorem $2.1 \delta$ is a derivation on $L$. Now we write $\Gamma=F-\delta$. Then we have

$$
\begin{aligned}
(a b a)^{\Gamma} & =(a b a)^{F-\delta} \\
& =(a b a)^{F}-(a b a)^{\delta} \\
& =\left(a^{F}-a^{\delta}\right) b a=a^{F-\delta} b a=a^{\Gamma} b a \text { for all } a, b \in L .
\end{aligned}
$$

And so, we have $(a b a)^{\Gamma}=a^{\Gamma} b a$ for all $a, b \in L$. In otherwords, $\Gamma$ is a Jordan triple left centralizer on $L$. Since $R$ is a 2-torsion free semiprime ring, one can conclude that $\Gamma$ is a Jordan left centralizer by Theorem 3.1. Hence $F$ is of the form $F=\Gamma+\delta$, where $\delta$ is a derivation and $\Gamma$ is a Jordan left centralizer on $L$. Hence $F$ is a generalized Jordan derivation on $L$.

THEOREM 3.3. Let $R$ be a 2-torsion free prime ring and $L \nsubseteq Z(R)$ be a square-closed Lie ideal. If $F: R \rightarrow R$ is a generalized Jordan triple derivation on $L$, then $F$ is a generalized derivation on $L$.

Proof. By Theorem 3.2 and Theorem of [2] we get the required result.

In conclusion, it is tempting to conjecture as follows:

CONJECTURE 3.1. Let $R$ be a 2-torsion free semiprime ring and $L \nsubseteq Z(R)$ be a Lie ideal of $R$. If $F: R \rightarrow R$ is a generalized Jordan triple derivation on $L$, then $F$ is a generalized derivation on $L$.

\section{REFERENCES}

[1] M. Ashraf - A. Ali - S. Ali, On Lie ideals and generalized $(\theta, \phi)$-derivations in prime rings, Comm. Algebra, 32 (2004), pp. 2877-2785.

[2] M. Ashraf - N. Rehman - S. Ali, On Lie ideals and Jordan generalized derivations of prime rings, Indian J. Pure and Appl. Math., 32 (2) (2003), pp. 291-294.

[3] M. Ashraf - N. Rehman, On Jordan generalized derivations in rings, Math. J. Okayama Univ., 42 (2000), pp. 7-9.

[4] J. BeRgen - I.N. HeRstein - J.W. KeR, Lie ideals and derivations of prime rings, J Algebra, 71 (1981), pp. 259-267. 
156 Motoshi Hongan - Nadeem ur Rehman - Radwan Mohammed AL-Omary

[5] M. Bresar, On the distance of the compositions of two derivations to the generalized derivations, Glasgow Math. J., 33 (1)(1991), pp. 89-93.

[6] M. BRESAR, Jordan mappings of semiprime rings, J. Algebra, 127 (1989), pp. $218-228$.

[7] M. Bresar, Jordan derivations on semiprime rings, Proc. Amer Math. Soc., 104 (1988), pp. 1003-1006.

[8] M. Bresar - J. Vukman, Jordan derivations on prime rings, Bull. Austral Math. Soc., 37 (1988), pp. 321-322.

[9] C. Chuang, GPI's having coefficients in Utumi Quotient rings, Proc. Amer Math. Soc., 103 (1988), pp. 723-728.

[10] J. M. Cusack, Jordan derivations on rings. Proc. Amer Math. Soc., 53 (1975), pp. 321-324.

[11] I. N. Herstein, Topics in Ring Theory. Chicago:Chicago Univ Press (1969).

[12] B. Hvala, Generalized derivations in rings, Comm. Algebra, 26 (1998), pp. 1149-1166.

[13] W. JING - S. LU, Generalized Jordan derivations on prime rings and standard opetaror algebras, Taiwanese J. Math., 7 (2003), pp. 605-613.

[14] C. LANski, Generalized derivations and nth power maps in rings. Comm. Algebra., 35 (2007), pp. 3660-3672.

[15] W. S. Martindale III, Prime ring satisfying a generalized polynomial identity, J. Algebra, 12 (1969), pp. 576-584.

[16] L. Molnar, On centralizers of an $H^{*}$-algebra, Publ. Math. Debrecen, 46, 1-2 (1995), pp. 89-95.

[17] N. Rehman - M. Hongan, Generalized Jordan derivations on Lie ideals associate with Hochschild 2-cocycles of rings. Rendiconti del Circolo Matematico di Palermo (to appear).

[18] J. Vukman, A note on generalized derivations of semiprime rings, Taiwanese J. Math., 11 (2007), pp. 367-370.

[19] J. Vukman - I. Kosi-UlbL, On centralizers of semiprime rings, Aequationes Math., 66 (2003), pp. 277-283.

[20] J. Vukman, Centralizers of semiprime rings, Comment Math. Univ. Carolinae, 42 (2) (2001), pp. 237-245.

[21] J. Vukman, An identity related to centralizers in semiprime rings, Comment Math. Univ. Carolinae, 40 (3) (1999), pp. 447-456.

[22] B. ZalaR, On centralizers of semiprime rings, Comment Math. Univ. Carolinae, 32 (1991), pp. 609-614.

Manoscritto pervenuto in redazione il 27 luglio 2010. 